\title{
Design and Implementation of Interference-Aware Channel Assignment Algorithm XiaoLiang Feng
}

\author{
Linyi City, Shandong Province. \\ 42189088@qq.com
}

Grade Nine Class 19, The first High School of Tancheng County,

Keywords: interference, channel assignment, wireless mesh network.

\begin{abstract}
This paper aims at maximizing system throughput by making use of multiple radios and multiple channels. This paper designs and implements a centralized channel assignment algorithm (IACA algorithm) in our Wireless Mesh Network test bed. This paper finds that IACA can improve system throughput by 5 to 6 times with each node using 2 radios and 12 channels.
\end{abstract}

\section{Introduction}

The availability of multiple channels defined in 802.11 standard provides possibility to increase system throughput by using Multiple Radios and Multiple Channels. It necessitates the researchers to design specific algorithm to make use of MRMC to improve the system throughput. In this paper, we aim at maximizing system throughput by making use of multiple radios and multiple channels. Channel Assignment is a basic issue for wireless network, and a lot of research works have been done in this area. However, most of previous works is evaluated using simulations, but we implement our algorithm in the real test bed, and find many practical problems during the experiment.

We design and implement a centralized channel assignment algorithm in our Wireless Mesh Network test bed. Following major contributions have been made in this paper. First, we design a centralized interference-aware channel assignment algorithm for 802.11 mesh network. To measure the interference extent for one channel from neighboring links, a new metric Interference Degree is proposed, referred to sum of traffic load in neighboring links working in the same channel. Based on the new metric, a centralized channel assignment algorithm is proposed to maximize the system throughput. Second, to make the Channel Assignment algorithm work in the real test bed, we design a algorithm based on channel assignment algorithm to collect topology information and distributed channel assignment result. Third, we did a lot of test to evaluate the performance of IACA in real test bed. According to the test results, compared with single radio single channel solution, we find that IACA can improve system throughput by 5 to 6 times with each node using 2 radios and 12 channels.

The result of this paper can be applied in cyber marine shipping. For example, we can utilize the multi-radio multi-channel technology to maximize the throughput of WMN in the local network of the marine ship.

\section{Design of Interference-Aware Channel Assignment Algorithm}

\subsection{Overview of Interference-Aware Channel Assignment protocol}

As we can see from following figure, IACA protocol is divided into three modules, namely topology generation module, Channel Assignment Algorithm part, and Channel Assignment Execution part. For the first module, its objective is to collect the topology information and the traffic information. For the second module, its main task is to calculate the Channel Assignment Result based on the topology information and the traffic load information. For the third module, its main purpose is to distributed the Channel Assignment result and execute the Channel Assignment command. 


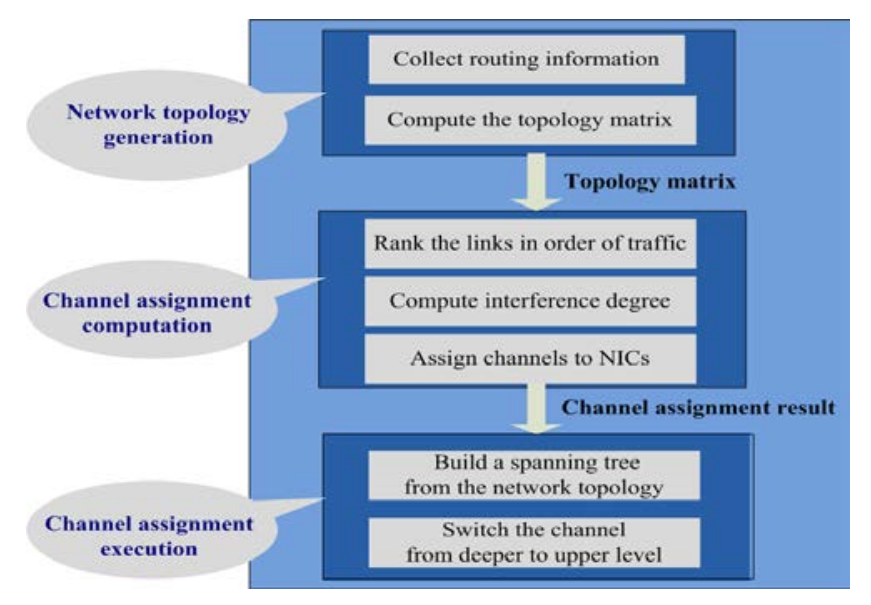

Fig 1. Channel assignment software architecture

\subsection{IACA centralized channel assignment algorithm}

To evaluate the channel condition, we design a new metric called "interference degree", I_d(channel k): The sum of traffic load that a link may experience on channel $\mathrm{k}$ in its interference region, which is mathematically defined as following. As we can see from Equitation (1), ${ }^{\operatorname{tr}\left(l_{j}\right)}{ }_{\text {is }}$ referred to the traffic load of link $l_{j}$, and ${ }^{\left|l_{i}-l_{j}\right| \leq 2}$ means that the hop distance between ${ }^{l_{i}}$ and ${ }^{l_{j}}$ is less than 2, ${ }^{\operatorname{ch}\left(l_{j}\right)=k}$ means that link ${ }^{l_{j}}$ works on channel k.

$$
I_{-} d_{l_{i}}(\text { channel } \mathrm{k})=\sum_{\substack{I_{i}-I_{j} \mid \leq 2 \\ \operatorname{ch}\left(l_{j}\right)=k}} \operatorname{tr}\left(l_{j}\right)
$$

We divide our algorithm into two steps. First, we rank the links in the decreasing order of link traffic so that the link with more traffic load will be firstly assigned. Second, we assign NICs and channels to the links. Using this metric, we will assign the channel with minimum interference degree to the link with maximum traffic load. To keep the network connectivity, we consider the channel assignment problem case-by-case. Based on number of available free radios for node pair of one link, we divide the problem into three cases. Free radio here refers to the radio that is not assigned to one link. In the first case, we assume that node pair of the link both has free radios. Here we denote the node pair by node_1 and node_2. As we can see from figure 2, node_1 and node_2 both have one free radio. We will select one free radio from node1, and one free radio from node2, and then assign any channel that has the least degree of interference to the virtual link.
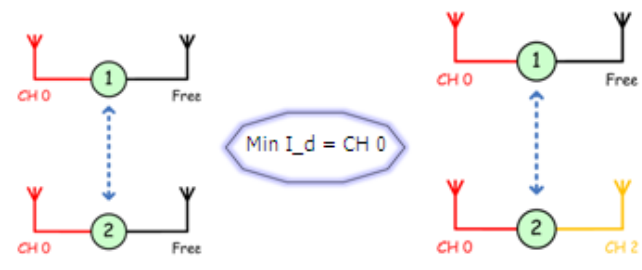

Fig 2. Channel assignment Case 1, both node_1 and node_2 have at least one free radio

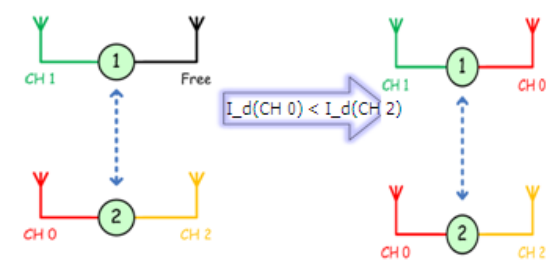

Fig 3. Channel assignment Case 2, One node has no free radios and the other node has free radio

In the second case, we assume that one node has no free radios and the other node has free radios. As we can see from figure 3, node 1 has one free radio, and node 2 has no free radio. Node 2 will select one radio, and the channel the radio works at will have least interference degree. The free radio of node 1 will switch to the same channel as the selected radio of node 2 , so the two radios will compose the link.

In the third case, we assume that both node 1 and node 2 have no free radios. To make the problem tractable, we further divide the problem into two sub-cases, case 3.a, and case 3.b. In case 
3.a, we assume that node 1 and node 2 have common channels while in case 3.b, node 1 and node 2 have no common channels. In case 3.a, since node 1 and node 2 both have one radio pair which works at the same channel, this radio pair will be selected as the link. If the two nodes have multiple radio pair which works at the same channel, we will the radio pair whose channel has the minimum interference degree. In case 3.b, because there is no radio pair which works at the same channel, we will have to merge some radios. We will select one radio from node 1 and one radio from node 2, and they will be assigned one channel with least interference degree. Other radios which are connected to the selected two radios will switch to the same channel.

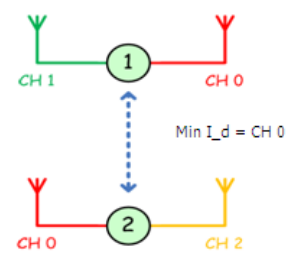

Fig 4. Channel assignment Case 3.a, Node_1 and node_2 have common channels

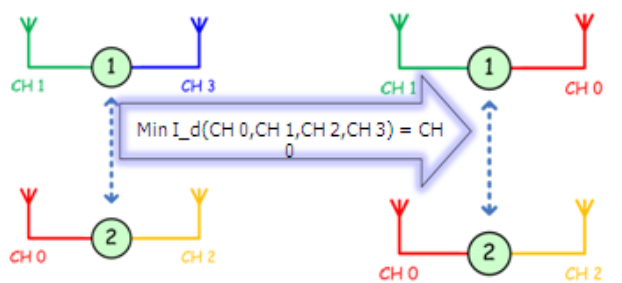

Fig 5. Channel assignment Case 3.b, Node_1 and node_2 have no common channels

The final result is stored in a data structure called, CA-Result matrix. As we can see from figure 6 , the row number denotes the node id, and the column number denotes the radio number. If the $j$ th radio of node $i$ and $j$ are assigned channel $c$, then CA-Result $[i][j]=c$ As we can see, the matrix is the channel assignment result of the network in which all nodes have two radios. The radio 0 of node 2 has been assigned channel 1 .

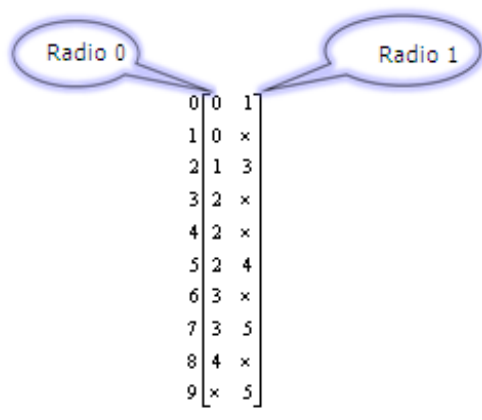

Fig 6. CA-Result matrix

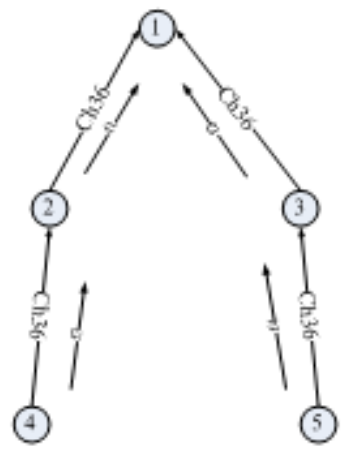

Fig 7. Network using single channel

\section{Performance Evaluation}

As we can see from figure 7 and figure 8, we set up a tree network of 5 nodes to compare the performance of network using centralized channel assignment algorithm and network without using centralized channel assignment algorithm. Each radio is equipped with 2 radios. We generate 4 single-hop flows in the network. As we can see from figure 7, all the radios use the same channel, channel 36 to transmit packets. As we can see from figure 8, radios are using different channels to communicate, such as channel 149, channel 36, channel 64, channel 44.

We use system throughput as our evaluation metrics. System throughput is defined as the sum of all traffic flow's data rate, which is mathematically defined as following.

$$
\text { System - throughput }=\sum r_{i}
$$

We compare system throughput of different traffic flows, including UDP traffic flows. As we can see from figure 9, the UDP system throughput of network using 4 channels is 5or 6 times larger than that of network using single channel. However, the UDP system throughput of network using 4 channels fluctuates a lot which means that the UDP system throughput is not stable. 


\section{Conclusion}

In this paper, we design and implement a centralized channel assignment algorithm. Following major contribution has been made in this paper. First, we design a centralized channel assignment algorithm. In this algorithm, interference relationship and traffic load are considered to maximize the system throughput. A metric, called interference degree, is proposed to evaluate the interference extent of single channel. Second, a complete algorithm is designed, including the collection of topology information, and the distribution of channel assignment result. Third, we implement the algorithm in our test bed, and test the performance of our algorithm. The test result shows that using 4 orthogonal channels and 2 radios, our algorithm can improve the UDP network system throughput about 5 to 6 times.

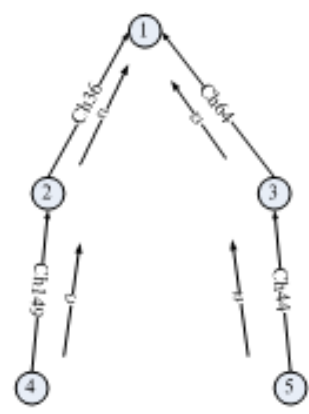

Fig8. Network using multiple channels

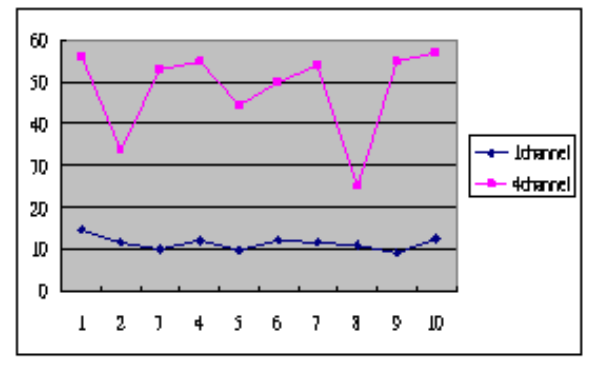

Fig9. UDP System throughput comparison

\section{References}

[1] A. P. Subramanian, M. M. Buddhikot, and S. C. Miller, "Interference Aware Routing in Multi-Radio WirelessMesh Networks,” IEEE Wksp. Wireless Mesh Networks, Sept. 2006, pp. 55-63.

[2] A. Raniwala and T.-C. Chiueh, "Architecture and Algorithms for an IEEE 802.11-Based Multi-Channel Wireless Mesh Network,” IEEE INFOCOM, Mar. 2005, pp. 2223-34.

[3] Arindam K.Das, Hamed M.K. Alazemi, Rajiv Vijayakumar, and Sumit Roy, "Optimization models of fixed channel assignment in wireless mesh networks with multiple radios”, SECON, 2005.

[4] Ashish Raniwala, Kartik Gopalan, and Tzi-cker Chiueh,“Centralized Channel Assignment and Routing Algorithms for Multi-Channel Wireless Mesh Networks,” Mobile Computing and Communications Review, vol. 8, no. 2, pp. 50-65, April 2004.

[5] “A new channel assignment strategy towards the wireless mesh networks", Procedings 10th Asia-Pacific Conference on Communications and the 5th International Symposium on Multi-Dimensional Mobile Communication, 2004. 